Mit Reisefieber ins Reisefieber ...

\title{
Malaria endet bei Senioren oft tödlich
}

\begin{abstract}
Das Risiko, nach einer Reise in ein Endemiegebiet an Malaria zu sterben, steigt mit zunehmendem Alter. Besonders hoch ist das Todesrisiko bei Reiserückkehrern aus Gambia.
\end{abstract}

In einer englischen Studie starben infizierte Senioren über 65 Jahre fast zehnmal so häufig an Malaria wie 18- bis 35-Jährige (4,6 vs. 0,5\%). An der Beobachtungsstudie waren knapp 40.000 Patienten beteiligt, die zwischen 1987 und 2006 gemeldet worden waren. 191 waren im Zusammenhang mit der Malaria-Erkrankung gestorben, 184 davon an einer Malaria tropica.

\section{Mangelndes Risikobewusstsein}

Todesfälle betrafen vor allem Touristen. Grund dafür: das mangelnde Risikobewusstsein. Afrikanischstämmige Patienten machten zwar das Gros der in England diagnostizierten Malariafälle aus; sie seien aber durch ihr Immunsystem besser geschützt, und sie suchten auch schneller ärztliche Hilfe, weil sie mit den frühen Symptomen vertraut sind und die Malaria als lebensbedrohliche Krankheit wahrnehmen. Insbesondere Gambia-Touristen denken seltener an die empfohlene Malariaprophylaxe und sind sich der möglichen Lebensgefahr oft nicht bewusst.

\section{Risiko steigt stetig}

Warum die Malaria mit zunehmendem Alter häufiger tödlich verläuft, bleibt unklar, da das Risiko offenbar schon von Kindesalter an stetig zunimmt.

Insbesondere Risikogruppen wie ältere Reisende sowie Reisende nach Gambia sollten auf die besondere Gefährdung hingewiesen werden. Mückenschutz sowie eine Chemoprophylaxe seien unerlässlich, ebenso wie die rasche Vorstellung beim Arzt im Falle einer fieberhaften Erkrankung nach Rückkehr aus einem Endemiegebiet.

(eo)

Checkley A M et al, BMJ 2012, 344 (online first)

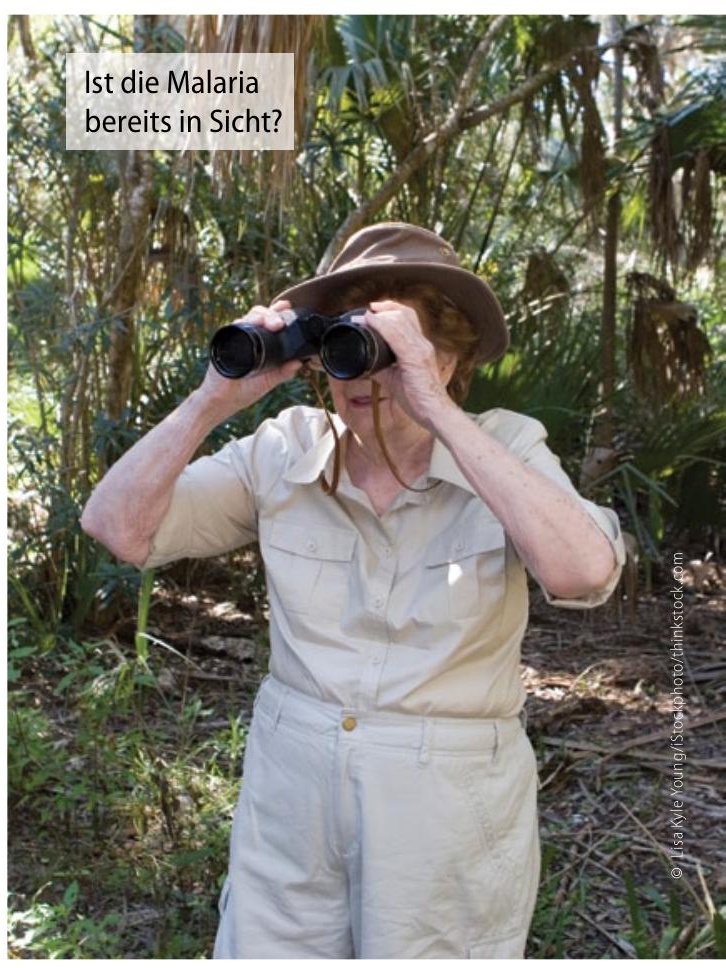

\section{Ob Thrombose oder Arthritis}

\section{Arthroskopie für alte Knie riskanter?}

\begin{abstract}
Arthroskopische Eingriffe am Knie resultieren offenbar auch bei Senioren relativ selten in schwerwiegenden Komplikationen wie Thrombembolie oder pyogener Arthritis.
\end{abstract}

Ein US-Orthopädenteam hat Daten von über 300.000 mindestens 65 Jahre alten Kniepatienten ausgewertet, die sich zwischen 2005 und 2008 einer Meniskektomie unterzogen hatten. In der Studie lag das Risiko für eine tiefe Beinvenenthrombose (TVT) bei 0,8\%, für eine Lungenembolie bei $0,2 \%$. Eine pyogene Arthritis entwickelten $0,4 \%$ der Patienten. Das relative
Risiko einer eitrigen Gelenkinfektion war bei den Männern deutlich höher als bei den Frauen. Letztere neigten dagegen eher zu einer Thrombembolie. Die Unterschiede waren jeweils statistisch signifikant.

\section{Kein erhöhtes Infektionsrisiko}

Die Studie zeigt, so die Autoren um Sharon L. Hame, dass ältere Patienten nach der Knie-Op. kein erhöhtes Infektionsrisiko zu befürchten haben. Für Patienten aller Altersklassen liegt es bei 0,12-0,42\%. Auch die Gefahr einer TVT oder Lungenembolie ist bei den Senioren offenbar nicht größer als bei jüngeren Patienten.
Es sei dennoch Wachsamkeit in Bezug auf klinische Warnzeichen angebracht, resümieren Hames und Kollegen. In Deutschland empfiehlt die S3-Leitlinie "Thromboseprophylaxe“ nach länger dauernden arthroskopischen Eingriffen an der unteren Extremität eine medikamentöse VTE-Prophylaxe „bis zum Erreichen der normalen Beweglichkeit mit einer Belastung von mindestens $20 \mathrm{~kg}$, mindestens aber für sieben Tage“.

Hame SL et al, Complications of Arthroscopic Meniscectomy in the Older Population. Am J Sports Med 2012, (online first) 